\title{
The implementation of natural language processing to extract index lesions from breast magnetic resonance imaging reports
}

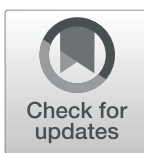

\author{
Yi Liu', Qing Liu², Chao Han'1, Xiaodong Zhang ${ }^{1}$ and Xiaoying Wang ${ }^{1 *}$ (D)
}

\begin{abstract}
Background: There are often multiple lesions in breast magnetic resonance imaging (MRI) reports and radiologists usually focus on describing the index lesion that is most crucial to clinicians in determining the management and prognosis of patients. Natural language processing (NLP) has been used for information extraction from mammography reports. However, few studies have investigated NLP in breast MRI data based on free-form text. The objective of the current study was to assess the validity of our NLP program to accurately extract index lesions and their corresponding imaging features from free-form text of breast MRI reports.

Methods: This cross-sectional study examined 1633 free-form text reports of breast MRls from 2014 to 2017. First, the NLP system was used to extract 9 features from all the lesions in the reports according to the Breast Imaging Reporting and Data System (BI-RADS) descriptors. Second, the index lesion was defined as the lesion with the largest number of imaging features. Third, we extracted the values of each imaging feature and the BI-RADS category from each index lesion. To evaluate the accuracy of our system, 478 reports were manually reviewed by two individuals. The time taken to extract data by NLP was compared with that by reviewers.
\end{abstract}

Results: The NLP system extracted 889 lesions from 478 reports. The mean number of imaging features per lesion was $6.5 \pm 2.1$ (range: 3-9; 95\% Cl: 6.362-6.638). The mean number of imaging features per index lesion was $8.0 \pm 1.1$ (range: 5-9; 95\% Cl: 7.901-8.099). The NLP system demonstrated a recall of 100.0\% and a precision of $99.6 \%$ for correct identification of the index lesion. The recall and precision of NLP to correctly extract the value of imaging features from the index lesions were 91.0 and $92.6 \%$, respectively. The recall and precision for the correct identification of the BI-RADS categories were 96.6 and $94.8 \%$, respectively. NLP generated the total results in less than $1 \mathrm{~s}$, whereas the manual reviewers averaged $4.47 \mathrm{~min}$ and $4.56 \mathrm{~min}$ per report.

Conclusions: Our NLP method successfully extracted the index lesion and its corresponding information from freeform text.

Keywords: BI-RADS, Breast cancer, Index lesion, Magnetic resonance imaging, Natural language processing, Rulebased method

\footnotetext{
*Correspondence: cjr.wangxiaoying@vip.163.com

'Department of Radiology, Peking University First Hospital, No. 8 Xishiku

Street, Xicheng District, Beijing 100034, China

Full list of author information is available at the end of the article
}

(c) The Author(s). 2019 Open Access This article is distributed under the terms of the Creative Commons Attribution 4.0 International License (http://creativecommons.org/licenses/by/4.0/), which permits unrestricted use, distribution, and reproduction in any medium, provided you give appropriate credit to the original author(s) and the source, provide a link to the Creative Commons license, and indicate if changes were made. The Creative Commons Public Domain Dedication waiver (http://creativecommons.org/publicdomain/zero/1.0/) applies to the data made available in this article, unless otherwise stated. 


\section{Background}

Breast cancer is one of the most commonly diagnosed types of cancer and a major global cause of cancer mortality in women, accounting for $23 \%$ of cancer diagnoses and $14 \%$ of cancer mortality each year [1]. Breast MRI can detect clinically and mammographically occult breast cancer in $3.1 \%$ of women with newly diagnosed breast cancer [2-5]. In 2007, the American Cancer Society issued a new clinical guideline recommending annual MRI screening for high-risk women [6-9].

Definitions of imaging features of breast MRI reports are clearly outlined in the Breast Imaging Reporting and Data System (BI-RADS) lexicon by the American College of Radiology [10]. BI-RADS descriptors have been used by radiologists in our department to describe breast lesion features and impression in breast MRI reports since 2013. This reduces variation between radiologists and standardizes the vocabulary used in MRI reports.

There are often multiple lesions in breast MRI reports and radiologists usually focus on describing the index lesion that is most crucial to clinicians in determining the management and prognosis of patients. Thus, identifying and extracting the index lesion is a critical clinical task. We assumed that the index lesion accounts for the largest number of imaging features and tried to extract the index lesions from free-form text by radiologists. While manual review may be feasible for small-scale studies, this method is very time-consuming, error-prone, and costly in studies with large sample sizes [11].

Natural language processing (NLP) consists of multiple steps, including a computer-based approach that converts free-form text into a standardized structured format with the help of lexicons and ontologies, ultimately creating standardized and normalized concepts. The structured information from reports could be acquired by NLP to determine index lesions, assuming all pertinent imaging features are recorded in the report [12]. NLP has been used for breast cancer diagnosis previously, including the extraction of BI-RADS categories and lesions from mammography reports [13, 14]. However, few studies have investigated NLP in breast MRI data based on free-form text. The objective of the current study was to assess the validity of our NLP program to accurately extract index lesions and their corresponding imaging features from free-form text of breast MRI reports using the rule-based method.

\section{Methods}

Data

This was a retrospective study that received approval from the responsible institutional review board of Peking University First Hospital with waiver of informed consent (2016[1178]). All breast MRI reports $(n=1633)$ which were written in Chinese from 2014 to 2017 were collected from Peking University First Hospital. 1633 patients were recruited into the study and each patient corresponded to one report. We used 1160 of the 1633 reports for development and refinement of the NLP system; the 478 not used for developing the system were selected and held out as an independent test set for the final evaluation of the NLP system. All patients met the following inclusion criteria: examination type was identified correctly and images were available for evaluation (T1- and T2-weighted fast spin-echo, diffusion-weighted imaging, echo planar imaging, short time inversion recovery, and dynamic contrast-enhanced T1-weighted fast spin-echo). Postoperative cases were excluded.

\section{BI-RADS MRI lexicon}

All imaging features and values were predefined based on the BI-RADS MRI lexicon [15] by two experienced experts in the field of breast imaging (Table 1). We also used RadLex, a controlled lexicon for radiology terminology, to identify semantic classes for terms in radiology reports. Our information extraction task focused on recognizing two semantic types of named entities: imaging features and their values. Imaging features are terms that refer to abnormalities in the breast and its attributes, such as mass, anatomic locations, size, shape, margin, signal, and enhancement kinetics. Imaging values are terms that modify imaging features to describe their features, f.e. "upper outer quadrant of the right breast" describes the location of a mass in the breast. BI-RADS categories include $0,1,2,3,4,5$, and 6 (Table 2).

\section{Preprocessing}

An internally developed NLP program (Smartree Clinical Information System, Beijing) was used to detect the index lesion and imaging features for each imaged breast from text reports (Fig. 1). This internally developed NLP program contained several analysis engines for various linguistics and clinical tasks, such as section segmentation, sentence segmentation and detection, tokenization, concept detection and normalization. In our pipeline, sequential processing modules were executed, producing an output of structured information of the index lesion. As shown in Fig. 2, each original breast MRI report spanned multiple paragraphs and occasionally split at various locations. The steps were as previously reported [16]. To facilitate NLP analysis, text reports were preprocessed. First, section boundaries were noted. This preprocessing segment input the original breast MRI report into sections. In the original breast MRI report, each section began with an anatomical location that was followed by another anatomical location (Example 1). Section segmentation was implemented as a predefined grammar consisting of a set of phases that annotate the section headers, that was, the anatomical location. 
Table 1 Imaging features and their value set to be extracted from breast MRI reports

\begin{tabular}{|c|c|c|c|c|}
\hline Entity type & Imaging features & Data type & Value set of imaging feature & Units \\
\hline \multirow[t]{10}{*}{ Mass } & Location & Categorical & Right, Left, Upper, Outer quadrant ... & N/A \\
\hline & Shape & Categorical & Oval, Round, Irregular ... & N/A \\
\hline & Size & Numerical & & $\mathrm{mm}$ \\
\hline & Signal & Categorical & & N/A \\
\hline & T1WI & Categorical & Low signal, Isointensity, High signal & N/A \\
\hline & $\mathrm{T} 2 \mathrm{Wl}$ & Categorical & Low signal, Isointensity, High signal & N/A \\
\hline & DWI & Categorical & Low signal, Isointensity, High signal & N/A \\
\hline & Margin & Categorical & Smooth, irregular, spiculated... & N/A \\
\hline & Internal enhancement & Categorical & Homogeneous, Heterogeneous ... & N/A \\
\hline & Enhancement kinetic curve & Categorical & Persistent, Plateau, Wash-out & N/A \\
\hline \multirow[t]{9}{*}{ NME } & Location & Categorical & Right, Left, Upper, Outer quadrant ... & N/A \\
\hline & Distribution pattern & Categorical & Focal area, Linear, Segmental ... & N/A \\
\hline & Scope & Numerical & & $\mathrm{mm}$ \\
\hline & Signal & Categorical & & N/A \\
\hline & T1WI & Categorical & Low signal, Isointensity, High signal & N/A \\
\hline & $\mathrm{T} 2 \mathrm{Wl}$ & Categorical & Low signal, Isointensity, High signal & N/A \\
\hline & DWI & Categorical & Low signal, Isointensity, High signal & N/A \\
\hline & Internal enhancement & Categorical & Homogeneous, Heterogeneous... & N/A \\
\hline & Enhancement kinetic curve & Categorical & Persistent, Plateau, Wash-out & N/A \\
\hline \multirow[t]{2}{*}{ Other associated findings } & Lymphadenopathy & Categorical & & N/A \\
\hline & Invasion of skin, nipple, chest wall, pectoralis muscle & Categorical & & N/A \\
\hline
\end{tabular}

T1WI T1-weighted imaging, T2WI T2-weighted imaging, DWI diffusion weighted imaging, NME non-mass enhancement, N/A not applicable

Second, the sentence boundaries were noted. Sentence segmentation was implemented using the predefined rule that used the periods as the marker word (Example 2). Third, our NLP program preprocessed the reports for imaging features tokenization based on our dictionary (Example 3). We developed the list of synonyms by inspecting a set of other 500 reports in addition to the predefined BI-RADS MRI lexicon. In addition, spelling mistakes were corrected and abbreviations were expanded to their full form. As shown in Figure 3, each breast MRI report was converted into a list of vocabulary.

\section{Example 1: Section segmentation.}

There is a $1.1 \mathrm{~cm} \times 1.0 \mathrm{~cm} \times 1.5 \mathrm{~cm}$ oval mass with irregular margin in [the upper inner quadrant of the left breast] location. Isointensity in T1WI, high signal in T2WI and high signal in DWI. There are dark internal septations in internal enhancement and kinetic curve is plateau. There is a non-mass enhancement in [the upper outer quadrant of the right breast] location. Isointensity in DWI, internal enhancement is heterogeneous.

Example 2: Sentence segmentation.

There is a $1.1 \mathrm{~cm} \times 1.0 \mathrm{~cm} \times 1.5 \mathrm{~cm}$ oval mass with irregular margin in the upper inner quadrant of the left breast [.] period Isointensity in T1WI, high signal in

Table 2 BI-RADS assessment categories and the number of index lesions found for each category

\begin{tabular}{lll}
\hline BI-RADS category & Implication & Number \\
\hline 0 & Requires additional imaging assessment and/or prior imaging for comparison & 0 \\
1 & Negative & 0 \\
2 & Benign discovery & 28 \\
3 & Probably benign discovery & 60 \\
4 & Suspected suspicious abnormality - biopsy & 87 \\
5 & Highly suggestive of malignancy - appropriate action should be taken & 119 \\
6 & Malignancy confirmed by biopsy - clinically feasible surgical resection & 177 \\
\hline
\end{tabular}




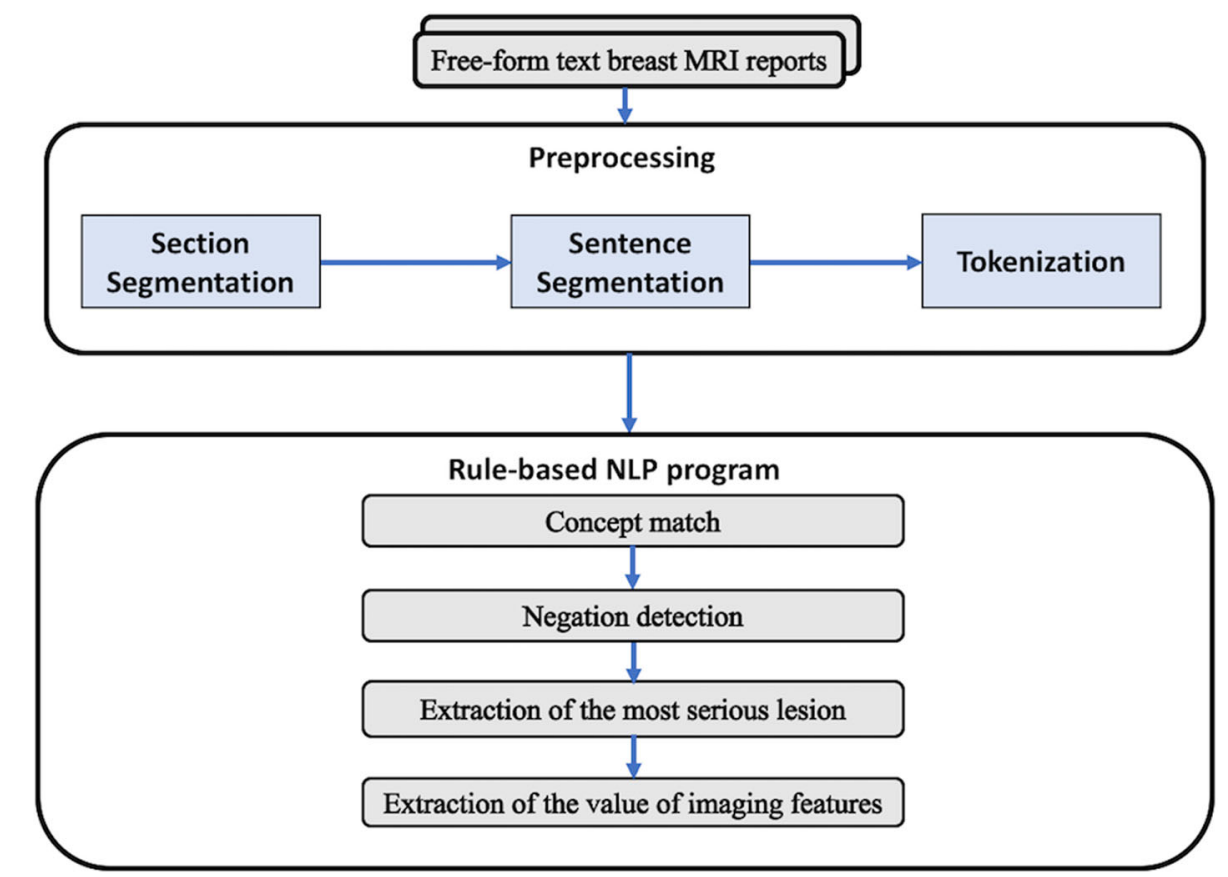

Fig. 1 An overview of our NLP program for extracting breast MRI reports

T2WI and high signal in DWI[.] period There are dark internal septations in internal enhancement and kinetic curve is plateau [.] period.

Example 3: Synonym conversion.

There is a $1.6 \mathrm{~cm} \times 1.2 \mathrm{~cm} \times 1.2 \mathrm{~cm}$ oval [mass] synonym: lesion with [smooth] synonym: clear boundary in the upper inner quadrant of the left breast.

Defining the index lesion by the number of imaging features.

The next step was concept match. Our processing module could match input features to BI-RADS terms by both exact match and synonym match. Then, we took the negation detection step. This step was to check the negated concepts. Distance and direction was both used to restrict the association between negation words and corresponding features. Three-word rule was imposed as the default distance for negative words after reviewing other 500 reports. We extracted negative words based on the given rule using our NLP tool.

We developed a strategy to compare the number of imaging features in each lesion, assuming that the index lesion accounts for the largest number of imaging features. We also selected the corresponding BI-RADS categories from which the highest score of each case was stored.

Extracting the value of imaging features from index lesions.

The last step was to extract the value of imaging features from index lesions and the accompanying signs (e.g. surrounding invasion or lymph node) as well as the

\begin{tabular}{lll}
\hline $\begin{array}{l}\text { Report } \\
\text { ID }\end{array}$ & Narrative \\
\hline 1 & $\begin{array}{l}\text { Imaging } \\
\text { description }\end{array}$ & $\begin{array}{l}\text { Bilateral breasts are almost entirely fat. Background parenchymal enhancement are } \\
\text { symmetric. } \\
\text { There is a } 1.1 \mathrm{~cm} \times 1.0 \mathrm{~cm} \times 1.5 \mathrm{~cm} \text { oval mass with irregular margin in the upper inner } \\
\text { quadrant of the left breast. Isointensity in T1WI, high signal in T2WI and high signal in } \\
\text { DWI. There are dark internal septations in internal enhancement and kinetic curve is } \\
\text { plateau. There is a non-mass enhancement in the upper outer quadrant of the right } \\
\text { breast. Isointensity in DWI, internal enhancement is heterogeneous. } \\
\text { There is no invasion of skin, nipple, chest wall and pectoralis muscle in bilateral breast. } \\
\text { There is no enlarged lymph node in bilateral armpit. }\end{array}$ \\
\hline 1 & $\begin{array}{l}\text { Bl-RADS category of the mass in upper outer quadrant of the right breast: } 6 . \\
\text { Bl-RADS category of the non-mass enhancement in the upper outer quadrant of the left } \\
\text { breast : } 3 .\end{array}$ \\
\hline impression
\end{tabular}

Fig. 2 A representative original breast MRI report. The report consists of an imaging description and diagnostic impression 


\begin{tabular}{|c|c|c|c|c|c|}
\hline Tokenized Text & Feature & Value & Section ID & Sentence ID & Feature ID \\
\hline Bilateral breasts & fibroglandular tissue & entirely fat & 1 & 1 & 1 \\
\hline Background parenchymal & background parenchymal enhancement & symmetric & 1 & 2 & 1 \\
\hline mass & mass & & & & \\
\hline $1.1 \mathrm{~cm} \times 1.0 \mathrm{~cm} \times 1.5 \mathrm{~cm}$ & size & $1.1 \mathrm{~cm} \times 1.0 \mathrm{~cm} \times 1.5 \mathrm{~cm}$ & 2 & 1 & 1 \\
\hline oval & shape & oval & 2 & 1 & 2 \\
\hline margin & margin & irregular & 2 & 1 & 3 \\
\hline \multirow[t]{2}{*}{ the upper inner quadrant of the left breast } & location & the upper inner quadrant of the left breast & 2 & 1 & 4 \\
\hline & signal & & & & \\
\hline T1WI & $\mathrm{T} 1 \mathrm{~W} \mid$ & isointensity & 2 & 1 & 5 \\
\hline $\mathrm{T} 2 \mathrm{WI}$ & $\mathrm{T} 2 \mathrm{WI}$ & high signal & 2 & 1 & 6 \\
\hline DWI & DWI & high signal & 2 & 1 & 7 \\
\hline internal enhancement & internal enhancement & dark internal septations & 2 & 1 & 8 \\
\hline kinetic curve & kinetic curve & plateau & 2 & 1 & 9 \\
\hline non-mass enhancement & non-mass enhancement & & & & \\
\hline $\begin{array}{l}\text { the upper outer quadrant of the right } \\
\text { breast }\end{array}$ & location & $\begin{array}{l}\text { the upper outer quadrant of the right } \\
\text { breast }\end{array}$ & 2 & 2 & 1 \\
\hline DWI & DWI & isointensity & 2 & 2 & 2 \\
\hline internal enhancement & internal enhancement & heterogeneous & 2 & 2 & 3 \\
\hline skin & skin & no invasion & 3 & 1 & 1 \\
\hline nipple & nipple & no invasion & 3 & 1 & 2 \\
\hline chest wall & chest wall & no invasion & 3 & 1 & 3 \\
\hline pectoralis muscle & pectoralis muscle & no invasion & 3 & 1 & 4 \\
\hline lymph node & lymph node & no enlarged & 3 & 2 & 1 \\
\hline
\end{tabular}

Fig. 3 Annotated text with the final extraction results. Each report was converted into a list of vocabulary flagged with its section, sentence, and vocabulary number after section segmentation, sentence segmentation, and tokenization

highest BI-RADS category as the final BI-RADS category for the index lesion. The final results are displayed in Fig. 3.

\section{Standard reference}

In order to evaluate the accuracy of our NLP system, 478 selected reports were reviewed by two diagnostic radiologists with 2 and 6 years of postgraduate experience. Two manual reviewers were blinded from the NLP results and extracted information independently. Any discrepancies between data found were evaluated by a third independent reviewer. Reports contained either no lesion $(n=7)$, one lesion $(n=93)$, or multiple lesions $(n=378)$. The diagnostic impression of the 7 reports with no lesion was BI-RADS 1 . The remaining 471 positive cases exhibited a total of 889 lesions containing 471 index lesions. The number of index lesions for each category is displayed in Table 2. The mean number of imaging features in an index lesion was $8.2 \pm 1.2$ (range: $5-9$; 95\% confidence interval (CI): 8.092-8.308). This dataset of 478 reports comprised the gold standard for our evaluation and comparative analyses were performed.

\section{Time consumption}

Each reviewer was asked to record the time required to extract the index lesion, corresponding imaging features, values, accompanying signs, and BI-RADS category from each report. Afterwards, we compared the time to data acquisition between the NLP program and the manual review.

\section{Statistical analysis}

The extraction and retrieval of information by the NLP program was compared to standard reference. The kappa coefficient was used to assess the agreement between two reviewers. Good inter-observer agreement was noted between two readers when $k \geq 0.60$. The mean number of imaging features per lesion and the mean number of imaging features per index lesion were described in the form of mean \pm standard deviation. To calculate overall performance metrics, results were combined into the $2 * 2$ table. Recall and precision was determined for the information collected by the NLP program, compared against the standard reference.

\section{Results}

\section{Inter-observer agreement}

In regard to the total number of lesions and index lesions, location, enhancement kinetic curve, nipple invasion, skin invasion, chest wall invasion, pectoralis muscle invasion and BI-RADS category of the index lesions, the inter-observer reliability showed almost perfect agreement, with $\mathrm{k}$ values of $0.92,0.94,0.83,0.90,0.89,0.88$, 0.90 .0 .91 and 0.93 , respectively. In regard to the shape, size, T1WI, T2WI, DWI and internal enhancement of the index lesions, there was substantial agreement, with 
a $\mathrm{k}$ value of $0.73,0.78,0.80,0.79,0.79$ and 0.78 , respectively. Any discrepancies between data found were evaluated by a third independent reviewer.

\section{Imaging features of lesions}

The NLP system extracted 889 lesions from 478 reports. Reports contained either no lesion $(n=7)$, one lesion $(n=93)$, two lesions $(n=338)$ or three lesions $(n=40)$.

The mean number of imaging features per lesion was $7.6 \pm 1.4$ (range: $5-9$; 95\% CI: 7.315-7.885) for the reports with one lesion. For the reports with 2 lesions, the mean number of imaging features per lesion of index lesion was $8.2 \pm 1.0$ (range: $5-9$; 95\% CI: 8.093-8.307), and that of non-index lesion was $4.5 \pm$ 1.3 (range: 3-8; 95\% CI: 4.361-4.639). For the reports with 3 lesions, the mean number of imaging features per lesion of index lesion was $8.5 \pm 0.7$ (range: 6-9; 95\% CI: 8.283-8.717), and that of non-index lesion was $5.2 \pm 1.6$ (range: $3-8$; 95\% CI: 4.849-5.551). The mean number of imaging features per lesion was $6.5 \pm 2.1$ (range: $3-9$; 95\% CI: 6.362-6.638) for all lesions and that was $8.0 \pm 1.1$ (range: 5-9; 95\% CI: 7.901-8.099) for index lesions extracted by the NLP system. The number of features in each lesion was different. The number of features in each lesion and the number of lesions in each report was displayed in Fig. 4.

NLP system performance during index lesion extraction The NLP system extracted 471 index lesions and 469 of them were true. The NLP system demonstrated a recall of $99.6 \%$ and a precision of $99.6 \%$ for correct identification of index lesions.
NLP system performance during extraction of the value of imaging features

The number of incorrectly extracted cases in each imaging feature was showed in Table 3. For all breast MRI reports, the NLP system demonstrated a recall of $91.0 \%$ and a precision of $92.6 \%$ for the correct identification of the value of imaging features from the index lesions. The recall and precision for the correct identification of the BI-RADS categories were 96.6 and $94.8 \%$, respectively. The performance of the NLP system for individual study types is displayed in Table 4.

\section{Efficiency}

We compared the time to data acquisition between the NLP program and manual review. The average time for each manual reviewer to extract the index lesion and the corresponding features was $4.47 \pm 0.85 \mathrm{~min}(95 \% \mathrm{CI}$ : 4.393-4.547) and $4.56 \pm 0.89 \mathrm{~min}$ (95\% CI: 4.480-4.640) per report, respectively, whereas NLP generated the total results in less than $1 \mathrm{~s}$.

\section{Discussion}

This present study was conducted in order to evaluate the implementation of our NLP program, which extracts index lesions and corresponding values of imaging features from MRI reports. Our results indicate that NLP can accurately identify the index lesion in each case. The recall and precision of our system for achieving perfect image information extraction were above $85.0 \%$.

Previous studies have been published on extracting information using various NLP systems [13, 16]. For example, Gao et al. (2015) developed a rule-based NLP system to extract four mammographic findings (mass, calcification, asymmetry, and architectural distortion), which are closely related to the increased risk of breast

The number of features in each lesion and the number of lesions in each report

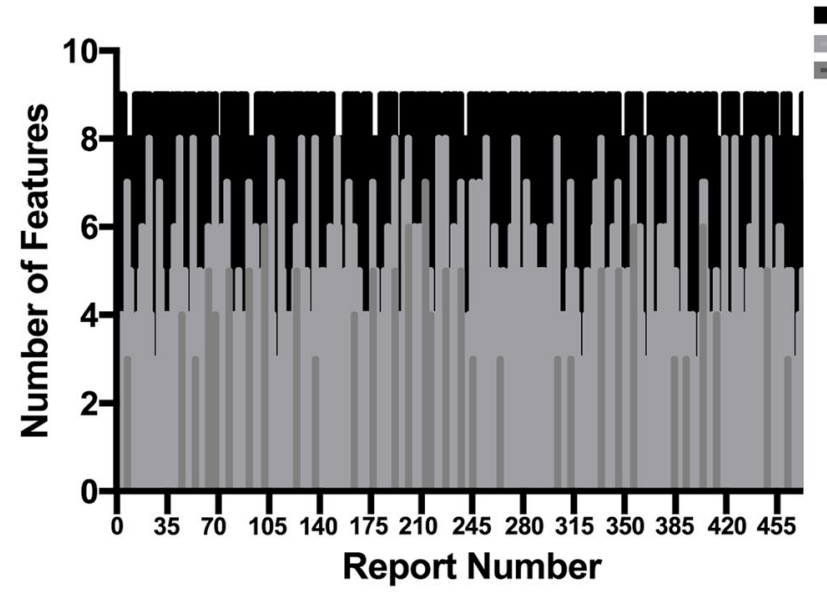

Index Lesion

Second Lesion

Third Lesion

\section{Report Number}

Fig. 4 The number of features in each lesion and the number of lesions in each report 
Table 3 Error analysis of the NLP system

\begin{tabular}{|c|c|c|c|}
\hline & Imaging features & Not detected (cases) & Not detected correctly (cases) \\
\hline \multirow[t]{15}{*}{ Index lesion } & Location & 16 & 2 \\
\hline & Shape & 2 & 24 \\
\hline & Size & 20 & 5 \\
\hline & T1Wl & 21 & - \\
\hline & $\mathrm{T} 2 \mathrm{Wl}$ & 18 & - \\
\hline & DWI & 12 & 10 \\
\hline & Margin & 7 & 9 \\
\hline & Internal enhancement & 20 & 3 \\
\hline & Enhancement kinetic curve & 20 & - \\
\hline & Lymphadenopathy & 12 & 20 \\
\hline & Nipple invasion & 29 & - \\
\hline & Skin invasion & 43 & - \\
\hline & Chest wall invasion & 5 & - \\
\hline & Pectoralis muscle invasion & 3 & - \\
\hline & BI-RADS category & 20 & 4 \\
\hline
\end{tabular}

NLP natural language processing, TIWI T1-weighted imaging, T2WI T2-weighted imaging, DWI diffusion weighted imaging, NME non-mass enhancement, BI-RADS Breast Imaging Reporting and Data System

cancer [16]. Jain et al. (1997) developed an NLP system (MedLEE) to encode the clinical information in mammogram reports to identify the suspected breast cancer [17]. Based on MedLEE, Sevenster et al. (2012) developed another NLP system, which could extract and correlate the findings and location [18]. However, those NLP system sometimes failed to extract the location, although the location phrases were added to the vocabulary. In addition, those studies used an NLP system to extract information from mammography reports, whereas few studies have addressed features/information extraction from breast MRI reports. Breast MRI is highly sensitive for detection and characterization of malignancy due to its great soft-tissue contrast capabilities. Index lesions are the most crucial factors, as they directly determine treatment and prognosis. Many retrospective studies require MRI information extracted from index lesions to analyze the association between lesion characteristics and clinical outcomes [19,20].

Our approach utilized a rule-based method that identified index lesions and corresponding features from breast MRI reports. Our rule-based system used concept match and negation detection that mapped imaging features in the free-form text reports to respective classes and achieved extremely high performance. According to our rule that index lesions account for the largest number of imaging features, the NLP automatically selected the index lesion from each report for a variety of efforts including follow-ups for breast cancer, targeted therapy, and lesion annotation. For example, the 10-year survival rate for breast cancer exceeded $70 \%$ and the survival rate for local lesions was about 89\% [21]. Therefore, follow- ups are very common for breast cancer patients. The purpose of follow-ups is to detect changes in index lesions in the short-term or assess the effects of treatment. If the NLP system is able to precisely extract and even compare the difference in index lesions at follow-ups, it will greatly eliminate the time-consuming process of manual extraction.

For all breast MRI reports in this study, NLP systems demonstrated a recall of $91.0 \%$ and a precision of $92.6 \%$ for the correct identification of the value of imaging features from the index lesions. The recall and precision for the correct identification of the BI-RADS categories were 96.6 and $94.8 \%$, respectively. The efficiency of the extraction of the value of each imaging feature was different. In addition to "Location", "Margin", and "Enhancement kinetic curve", the precision and recall of the values of other features were above 95 and $90 \%$, respectively, which is similar to the results of previous studies on extraction of image report information [22, 23]. The reasons for extraction errors included features were not detected or they were not detected correctly by the NLP tools. The main error was that features were not detected by our tools. Most of that errors resulted from the poor compliance with the BI-RADS lexicon. Our NLP system extracted features and values of features based on the BI-RADS lexicon. Although radiologists in our department were asked to describe breast lesion features and impression using BI-RADS descriptors after 2013, not all radiologists necessarily comply to these standards, which hinders the application of NLP. For example, as to the description of lesions, various expressions such as "a low-enhancement zone of necrosis in the 
Table 4 Accuracy in extracting complete descriptions of breast lesions by the NLP system

\begin{tabular}{|c|c|c|c|}
\hline Entity type & Imaging features & Recall & Precision \\
\hline \multirow[t]{9}{*}{ Mass } & Location & $90.1 \%$ & $95.7 \%$ \\
\hline & Shape & $85.4 \%$ & $94.1 \%$ \\
\hline & Size & $90.4 \%$ & $94.2 \%$ \\
\hline & T1WI & $90.3 \%$ & $94.1 \%$ \\
\hline & $\mathrm{T} 2 \mathrm{Wl}$ & $89.6 \%$ & $94.1 \%$ \\
\hline & DWI & $88.7 \%$ & $93.2 \%$ \\
\hline & Margin & $90.9 \%$ & $95.9 \%$ \\
\hline & Internal enhancement & $91.6 \%$ & $90.2 \%$ \\
\hline & Enhancement kinetic curve & $91.6 \%$ & $95.7 \%$ \\
\hline \multirow[t]{8}{*}{ NME } & Location & $90.9 \%$ & $95.2 \%$ \\
\hline & Distribution pattern & $86.2 \%$ & $94.1 \%$ \\
\hline & Scope & $89.2 \%$ & $92.3 \%$ \\
\hline & T1WI & $91.1 \%$ & $93.6 \%$ \\
\hline & $\mathrm{T} 2 \mathrm{Wl}$ & $88.9 \%$ & $93.2 \%$ \\
\hline & DWI & $88.6 \%$ & $94.0 \%$ \\
\hline & Internal enhancement & $91.3 \%$ & $91.5 \%$ \\
\hline & Enhancement kinetic curve & $90.9 \%$ & $95.0 \%$ \\
\hline Lymphadenopathy & & $98.7 \%$ & $87.7 \%$ \\
\hline \multirow[t]{4}{*}{ Invasion } & Nipple & $98.6 \%$ & $86.4 \%$ \\
\hline & Skin & $97.4 \%$ & $86.8 \%$ \\
\hline & Chest wall & $97.7 \%$ & $86.9 \%$ \\
\hline & Pectoralis muscle & $96.2 \%$ & $85.8 \%$ \\
\hline BI-RADS category & & $96.6 \%$ & $94.8 \%$ \\
\hline Overall & & $91.5 \%$ & $92.9 \%$ \\
\hline
\end{tabular}

NLP natural language processing, T1WI T1-weighted imaging, T2WI T2weighted imaging, DWI diffusion weighted imaging, NME non-mass enhancement, BI-RADS Breast Imaging Reporting and Data System

center and rim homogeneous enhancement in the surrounding area" appear in the report; however NLP system cannot extract this as "rim enhancement" or "homogeneous enhancement". Another reason was that there were some spelling mistakes in our reports. Although spelling corrections were conducted during data preprocessing, our dictionary was not comprehensive and some infrequent spelling mistakes were not included. For example, the word "Bi-RAS" was used in the imaging diagnosis: "Bi-RAS category of the mass in the upper inner quadrant of the right breast: 6". The NLP system could not discern that "BI-RADS" was " 6 ". In clinical practice, incomplete or incorrect extraction information will affect the further analysis and utilization of clinical data. Error analysis showed that the extraction errors would be significantly reduced by augmenting our dictionary in the future.

Although the inter-observer variability of manual extraction was high, there were some discrepancies between two reviewers during information extraction. Our approach utilized a rule-based method that identified index lesions and corresponding features from breast MRI reports. As long as the ruled were clearly defined, key information could be accurately extracted from the free-form text reports. Our rule-based NLP method could efficiently avoid the discrepancies between reviewers during the manual extraction process, which was conductive to the mining of free-form text reports in clinical practice.

One of the most notable advantages of NLP was that it could complete all information extraction in a matter of seconds compared to the dozens of hours it would take for the manual reviewer to obtain the same information. This difference in time becomes more apparent when dealing with an increasing number of cases, and manual reviewers with time are prone to fatigue and therefore reduced efficiency and accuracy. The NLP program could thus significantly reduce the time and achieve this with high accuracy.

The literature suggests that health information technology could improve the efficiency and quality of health care [24], and NLP is an informatics tool that can help fulfill this promise. More specifically, the extracted information from the NLP system could be used to build cohorts for cancer studies [25-27]. To date, building cohorts for cancer studies has relied on the laborious and time-consuming manual selection of cancer cases. By extracting features of cancer automatically with the NLP system, the efficiency of cancer research could be improved. Some studies have selected patients with various conditions, including renal cysts [25], adrenal nodules [26], or specific BI-RADS assessment categories [27]. In addition to extracting the BI-RADS categories, our study also extracted lesion features and the value of each feature from breast MRI reports. Furthermore, in recent years, much artificial intelligence or computeraided diagnostic research used the information of reports to label lesions or organs. The annotation of imaging features is important and the NLP system facilitates the extraction of imaging features that can be directly used to label lesions or organs. Forsberg et al. (2017) used the clinically provided spine label annotations stored in an institution image archive as training data for deep learning-based vertebral detection [28]. The results demonstrated that clinically annotated image data from one image archive is sufficient to train a deep learning-based pipeline for accurate detection and labeling of MR images depicting the spine. Similarly, it is feasible to use annotated image data to train other disease prediction models, and simultaneous application of the NLP system to automatically extract annotation data will greatly increase the efficiency of this process.

There are some limitations to our NLP system, such as the selection of reports that are predefined for index 
lesions. First, the subject of our study were breast MRI free-form text reports using BI-RADS descriptors. Although our NLP system performed well on these reports, it was not suitable for other reports that did not use BI-RADS descriptors. Second, only 7 out of 478 cases reported no lesion in our data and there is no case with BI-RADS 0 or 1 in the rest of 471 reports which reflected the general population who underwent breast MRI. However, the number of negative cases was too small in our study which might impact on the method performance. We would add more negative cases to verify the performance of our NLP method in the future. In addition, the NLP system extracted the index lesion based on our hypothesis that the index lesion accounts for the largest number of imaging features. This hypothesis was artificially defined and was not the direct extraction of the index lesion. In the future, the index lesions should be directly extracted from images based on the image features.

\section{Conclusions}

The imaging features of index lesions in breast MRI can be extracted using our NLP system, which can be used to build cohorts for cancer studies and label data automatically for model training. In the future, data from structured reports could be used for NLP research and the efficiency of NLP could be further improved with enhancements of data quality.

\section{Abbreviations}

BI-RADS: Breast Imaging Reporting and Data System; MRI: magnetic resonance imaging; NLP: natural language processing

\section{Acknowledgements}

The authors would like to thank Changzheng He for providing guidance for the implementation of the natural language processing software tool. The authors assume full responsibility for database creation, analyses, and interpretation of the data.

\section{Availability of data and material}

The datasets generated and/or analysed during the current study are not publicly available due [Research institutions do not allow data uploads] but are available from the corresponding author on reasonable request.

\section{Author's contributions}

YL and XYW designed and implemented the approach, generated the data and wrote the draft of the manuscript. YL, QL, CH and XDZ set the goals, analyzed the data, and wrote the manuscript. All authors read and approved the final manuscript.

\section{Funding}

This research did not receive any specific grant from funding agencies in the public, commercial, or not-for-profit sectors.

\section{Ethics approval and consent to participate}

This was a retrospective study that received approval from the responsible institutional review board of Peking University First Hospital with waiver of informed consent (No: 2016[1178]).

\section{Consent for publication}

Not applicable.

\section{Competing interests}

The authors declare that they have no competing interests.

\section{Author details}

'Department of Radiology, Peking University First Hospital, No. 8 Xishiku Street, Xicheng District, Beijing 100034, China. 'Department of Radiolog, Peking University Cancer Hospital and Institute, No. 52 Fucheng Road, Haidian District, Beijing, China.

Received: 23 July 2019 Accepted: 25 November 2019

Published online: 30 December 2019

\section{References}

1. Jemal A, Bray F, Center MM, Ferlay J, Ward E, Forman D. Global cancer statistics. CA Cancer J Clin. 2011;61:69-90. https://doi.org/10.3322/caac. 20107.

2. Tillman GF, Orel SG, Schnall MD, Schultz DJ, Tan JE, Solin LJ. Effect of breast magnetic resonance imaging on the clinical management of women with early-stage breast carcinoma. J Clin Oncol. 2002;20(16):3413-23. https://doi. org/10.1200/JCO.2002.08.600

3. Bedrosian I, Mick R, Orel SG, Schnall M, Reynolds C, Spitz FR, et al. Changes in the surgical management of patients with breast carcinoma based on preoperative magnetic resonance imaging. Cancer. 2003;98(3):468-73. https://doi.org/10.1002/cncr.11490

4. Hylton N. Magnetic resonance imaging of the breast: opportunities to improve breast cancer management. J Clin Oncol. 2005;23(8):1678-84. https://doi.org/10.1200/JCO.2005.12.002.

5. Braun M, Pölcher M, Schrading S, Zivanovic O, Kowalski T, Flucke U, et al. Influence of preoperative MRI on the surgical management of patients with operable breast cancer. Breast Cancer Res Treat. 2008;111(1):179-87. https:// doi.org/10.1007/s10549-007-9767-5.

6. Beatty JD, Porter BA. Contrast-enhanced breast magnetic resonance imaging: the surgical perspective. Am J Surg. 2007;193(5):600-5. https://doi. org/10.1016/j.amjsurg.2007.01.015.

7. Schelfout K, Van Goethem M, Kersschot E, Colpaert C, Schelfhout AM, Leyman P, et al. Contrast-enhanced MR imaging of breast lesions and effect on treatment. Eur J Surg Oncol. 2004;30:501-7. https://doi.org/10.1016/j.ejso. 2004.02.003.

8. Zhang Y, Fukatsu H, Naganawa S, Satake H, Sato Y, Ohiwa M, et al. The role of contrast-enhanced MR mammography for determining candidates for breast conservation surgery. Breast Cancer. 2002;9(3):231-9.

9. Esserman L, Hylton N, Yassa L, Barclay J, Frankel S, Sickles E. Utility of magnetic resonance imaging in the management of breast cancer: evidence for improved preoperative staging. J Clin Oncol. 1999;17(1):110-9. https://doi.org/10.1200/JCO.1999.17.1.110.

10. Burnside ES, Sickles EA, Bassett LW, Rubin DL, Lee CH, lkeda DM, et al. The ACR BI-RADS experience: learning from history. J Am Coll Radiol. 2009;6(12): 851-60. https://doi.org/10.1016/j.jacr.2009.07.023.

11. Meystre SM, Savova GK, Kipper-Schuler KC, Hurdle JF. Extracting information from textual documents in the electronic health record: a review of recent research. Yearb Med Inform. 2008;1:128-44.

12. Demner-Fushman D, Chapman WW, McDonald CJ. What can natural language processing do for clinical decision support? J Biomed Inform. 2009:42:760-72. https://doi.org/10.1016/j.jbi.2009.08.007.

13. Bozkurt S, Lipson JA, Senol U, Rubin DL. Automatic abstraction of imaging features with their characteristics from mammography reports. J Am Med Inform Assoc. 2015;22:81-92. https://doi.org/10.1136/amiajnl-2014-003009.

14. Cai T, Giannopoulos AA, Yu S, Kelil T, Ripley B, Kumamaru KK, et al. Natural language processing technologies in radiology research and clinical applications. Radiographics. 2016;36(1):76-91. https://doi.org/10.1148/rg. 2016150080.

15. Nadkarni PM, Ohno-Machado L, Chapman WW. Natural language processing: an introduction. J Am Med Inform Assoc. 2011;18:544-51. https://doi.org/10.1136/amiajnl-2011-000464.

16. Gao H, Aiello Bowles EJ, Carrel D, Buist DS. Using natural language processing to extract mammographic findings. J Biomed Inform. 2015; 54:77-84.

17. Jain NL, Friedman C. Identification of findings suspicious for breast cancer based on natural language processing of mammogram reports. Proc AMIA Annu Fall Symp. 1997:829-33. 
18. Sevenster M, van Ommering R, Qian Y. Automatically correlating clinical findings and body locations in radiology reports using MedLEE. J Digit Imaging. 2012;25:240-9. https://doi.org/10.1007/s10278-011-9411-0.

19. Smitherman E, Hernandez A, Stavinoha PL, Huang R, Kernie SG, Diaz-Arrastia $R$, Miles DK. Predicting outcomes after pediatric traumatic brain injury by early magnetic resonance imaging lesion location and volume. J Neurotrauma. 2016 1;33(1):35-48.

20. Liu D, Scalzo F, Starkman S, Rao NM, Hinman JD, Kim D, et al. DWl lesion patterns predict outcome in stroke patients with thrombolysis. Cerebrovasc Dis. 2015;40(5-6):279-85.

21. Allemani C, Minicozzi P, Berrino F, Bastiaannet E, Gavin A, Galceran J, et al. Predictions of survival up to 10 years after diagnosis for European women with breast cancer in 2000-2002. Int J Cancer. 2013 May 15;132(10):2404-12.

22. Hripcsak G, Austin JH, Alderson PO, Friedman C. Use of natural language processing to translate clinical information from a database of 889,921 chest radiographic reports. Radiology. 2002;224(1):157-63. https://doi.org/10.1148/ radiol.2241011118

23. Dreyer KJ, Kalra MK, Maher MM, Hurier AM, Asfaw BA, Schultz T, et al. Application of recently developed computer algorithm for automatic classification of unstructured radiology reports: validation study. Radiology. 2005;234(2):323-9. https://doi.org/10.1148/radiol.2341040049.

24. Chaudhry B, Wang J, Wu S, Maglione M, Mojica W, Roth E, et al. Systematic review: impact of health information technology on quality, efficiency, and costs of medical care. Ann Intern Med. 2006;144(10):742-52.

25. O'Connor SD, Silverman SG, Ip IK, Maehara CK, Khorasani R. Simple cystappearing renal masses at unenhanced $\mathrm{CT}$ : can they be presumed to be benign? Radiology. 2013;269(3):793-800. https://doi.org/10.1148/ radiol.13122633.

26. Zopf JJ, Langer JM, Boonn WW, Kim W, Zafar HM. Development of automated detection of radiology reports citing adrenal finding. J Digit Imaging. 2012;25(1):43-9. https://doi.org/10.1007/s10278-011-9425-7.

27. Percha B, Nassif H, Lipson J, Burnside E, Rubin D. Automatic classification of mammography reports by BI-RADS breast tissue composition class. J Am Med Inform Assoc. 2012;19(5):913-6. https:// doi.org/10.1136/amiajnl-2011-000607.

28. Forsberg D, Sjoblom E, Sunshine JL. Detection and labeling of vertebrae in MR images using deep learning with clinical annotations as training data. J Digit Imaging. 2017;30(4):406-12. https://doi.org/10.1007/s10278-017-9945-X.

\section{Publisher's Note}

Springer Nature remains neutral with regard to jurisdictional claims in published maps and institutional affiliations.

Ready to submit your research? Choose BMC and benefit from:

- fast, convenient online submission

- thorough peer review by experienced researchers in your field

- rapid publication on acceptance

- support for research data, including large and complex data types

- gold Open Access which fosters wider collaboration and increased citations

- maximum visibility for your research: over $100 \mathrm{M}$ website views per year

At BMC, research is always in progress.

Learn more biomedcentral.com/submissions 